\title{
Knowledge, Practice of Personal Hygiene, School Sanitation, and Risk Factors of Contracting Diarrhea among Rural Students from Five Western Provinces in China
}

\author{
Yu-E Cha ${ }^{1,2}$, Yuan-Zheng Fu ${ }^{2,3}$ and Wei Yao ${ }^{1, *}$ \\ 1 National Center for Rural Water Supply Technical Guidance, Chinese Center for Disease Control and \\ Prevention, Beijing 102200, China; chayue@nieh.chinacdc.cn \\ 2 China CDC Key Laboratory of Environment and Population Health, National Institute of Environmental \\ Health, Chinese Center for Disease Control and Prevention, Beijing 100021, China; 18040028047@163.com \\ 3 Department of Toxicology, School of Public Health, China Medical University, Shenyang 110122, China \\ * Correspondence: yaowei@ncrwstg.chinacdc.cn; Tel.: +86-10-6971-3472; Fax: +86-10-8970-6594
}

\section{check for}

updates

Citation: Cha, Y.-E.; Fu, Y.-Z.; Yao, W. Knowledge, Practice of Personal Hygiene, School Sanitation, and Risk Factors of Contracting Diarrhea among Rural Students from Five Western Provinces in China. Int. J. Environ. Res. Public Health 2021, 18, 9505. https://doi.org/10.3390/ ijerph18189505

Academic Editors: Zhaomin Dong, Ying Wang and Xiaomin Li

Received: 27 July 2021

Accepted: 6 September 2021

Published: 9 September 2021

Publisher's Note: MDPI stays neutral with regard to jurisdictional claims in published maps and institutional affiliations.

Copyright: (c) 2021 by the authors. Licensee MDPI, Basel, Switzerland. This article is an open access article distributed under the terms and conditions of the Creative Commons Attribution (CC BY) license (https:// creativecommons.org/licenses/by/ $4.0 /)$.
Abstract: Background: Diarrhea is a global public health issue and a leading cause of childhood malnutrition, growth disturbances, and mortality. The spread of diarrhea is closely linked to the knowledge and maintenance of personal hygiene and quality of drinking water and sanitation facilities. However, there are few such investigations and analysis in rural areas of China. This study aims to determine the association between the risk of contracting diarrhea and knowledge and practices of personal hygiene and school sanitation among rural students as well as provide a scientific basis for preventing the spread of diarrhea and other infectious diseases. A stratified cluster sampling method was used to randomly select 12 rural primary schools in each of 5 counties where the Water, Sanitation and Hygiene (WASH) Plus Program has been implemented. The counties are located in the Guangxi Zhuang autonomous region, Chongqing municipality, Guizhou province, Yunnan province, and Xinjiang Uygur autonomous region. A single fourth-grade class was randomly chosen from each of the 60 schools for observation and a questionnaire survey. The study involved a total of 2330 students. The logistic regression method was adopted to determine the factors contributing to diarrhea in rural students. The results show that male students accounted for $49.40 \%(\mathrm{n}=1151)$ of the 2330 research subjects; the average age of the students was $9.9 \pm 0.3$ years. Approximately $33.09 \%$ of the students suffered from diarrhea in the three months leading up to the survey. The odds ratios (ORs) of students who did not know that "diarrhea can be prevented by washing fruits before eating them raw and not drinking untreated water" (OR: 1.303, 95\% confidence interval [CI]: $1.063,1.597)$ and that "the disease can be prevented by washing hands before meals and after going to the toilet" (OR: 1.522, 95\% CI: 1.207, 1.920) were higher than those who knew the above stated facts. Students who "have drunk untreated water at school" (OR: 1.584, 95\% CI: 1.268, 1.978), "have drunk untreated water at home" (OR: 1.643, 95\% CI: 1.319, 2.048), and "did not wash hands before every meal" (OR: 1.490, 95\% CI: 1.120, 1.983) were at a higher risk of contracting diarrhea than those who drank treated water at school and at home and washed their hands before every meal. Diarrhea was more likely to affect students who attended schools with unclean and poorly maintained toilets (OR: 1.586, 95\% CI: 1.261, 1.995) or toilets with flies (OR: 1.383, 95\% CI: 1.114, 1.717) and without adequate drinking water facilities (OR: 1.407, 95\% CI: 1.009, 1.962). The knowledge of methods to maintain personal hygiene, general hygiene practices, and school sanitation are the three major risk factors that account for the spread of diarrhea among rural students from five western provinces (municipalities and autonomous regions) of China. Therefore, to prevent such diseases and maintain health, it is important to provide students with health education, help them develop good hygiene habits, ensure the provision of clean water at schools, and improve the overall school environments.

Keywords: students; sanitation; rural students; knowledge of personal hygiene; practices of personal hygiene; diarrhea 


\section{Introduction}

Diarrhea is defined as a condition in which the patient passes loose or watery stools three or more times per day. It is known to occur commonly in children [1]. In developing and developed countries, diarrhea is mainly caused by viruses, bacteria, and parasites that are transmitted via contaminated food and water [2]. Diarrhea is a global public health issue and a leading cause of childhood malnutrition, growth disturbances, and mortality [3]. Diarrhea has been reported to be the eighth leading cause of mortality in the world, accounting for more than 1.66 million deaths in 2016. Most of the children who succumb to this disease are under the age of 5 years; diarrhea has led to the deaths of 446,000 children in this age group [4]. The incidence and mortality rates of diarrhea remain high among school-aged children, particularly those from low-income countries and regions. Every year, more than 2.8 billion school-aged children suffer from the disease [5]. In China, approximately 836 million people contract infectious diarrhea annually, of whom 678 million are children between 2 and 14 years of age [6]. Diarrhea is among the top five diseases in China with respect to either the mortality rate or disease burden measured in terms of disability-adjusted life years [7]. Schools are densely populated places and provide high-risk environments that facilitate the spread and outbreak of infectious diseases. Research shows that $>70 \%$ of China's public health emergencies occur in schools and $>80 \%$ of school emergencies are triggered by infectious diseases [8]. Zhang et al. [9,10] found a high relative risk of diarrheal disease in western China.

The spread of diarrhea is closely linked to the knowledge and maintenance of personal hygiene and quality of drinking water and sanitation facilities $[4,11,12]$. For example, washing hands with soap can reduce the risk of the disease by $42-47 \%$ and prevent close to 1 million diarrhea-induced deaths [13]. The provision of safe drinking water can further reduce the risk of developing diarrhea by $61 \%$; the provision of adequate sanitation facilities can reduce this risk by an additional $25 \%$ [14]. Wang et al. [6] reported a large gap between low-income countries and high-income countries in terms of the burden of diarrhea, which is mainly calculated in low-income countries based on the number of premature deaths and loss of the ability to work. Globalization has drastically increased the frequency of movement of people across the world. Therefore, a global effort is needed to curb the increasing burden of diarrhea by determining its risk factors and controlling its spread, along with carrying out the method of health literacy co-design in rural areas to improve health and equity of rural students [15]. Between 2016 and 2020, the Ministry of Education of the People's Republic of China and the United Nations International Children's Emergency Fund (UNICEF) implemented the WASH Plus Program (hereafter referred to as "the Program") at 300 rural primary schools in five provinces (municipalities or autonomous regions) in underdeveloped regions of western China. The Program aimed to provide safe drinking water and improve sanitation facilities (toilets and hand washing facilities) for children at the selected schools, thus helping them develop good hygiene practices [16]. Based on cross-sectional survey data from the Program obtained in 2018, we analyzed the associations between the contraction of diarrhea and students' knowledge and practice of personal hygiene and school sanitation conditions.

\section{Methods}

\subsection{Respondents}

A stratified cluster sampling method was used to randomly select five counties (one county in each of the five provinces (municipalities or autonomous regions), i.e., Guangxi Zhuang autonomous region, Chongqing municipality, Guizhou province, Yunnan province, and Xinjiang Uygur autonomous region) where the Program was implemented. These regions are highlighted in Figure 1.

The researchers randomly selected a single fourth-grade class from each of the 60 primary schools in the five counties (12 schools in each county). If a chosen class had $<30$ students, fifth-grade students were randomly selected to meet the quota requirement. A total of 2330 students participated in the survey. We use this formula $\mathrm{t} n=\frac{\mu_{\alpha}^{2} \times \pi \times(1-\pi)}{\delta^{2}} \mathrm{o}$ to 
calculate the sample size (n): $\alpha=0.05, \mu_{\alpha}=1.96, \pi$ is incidence rate of diarrhea is $10 \%$, and the error rate is $\left.3 \% . \delta^{2}=3 \% \times 3 \%=0.0009\right)$, the lost follow-up rate was $5 \%$, and the sample size was about 405 . Each province surveyed at least 405 students.

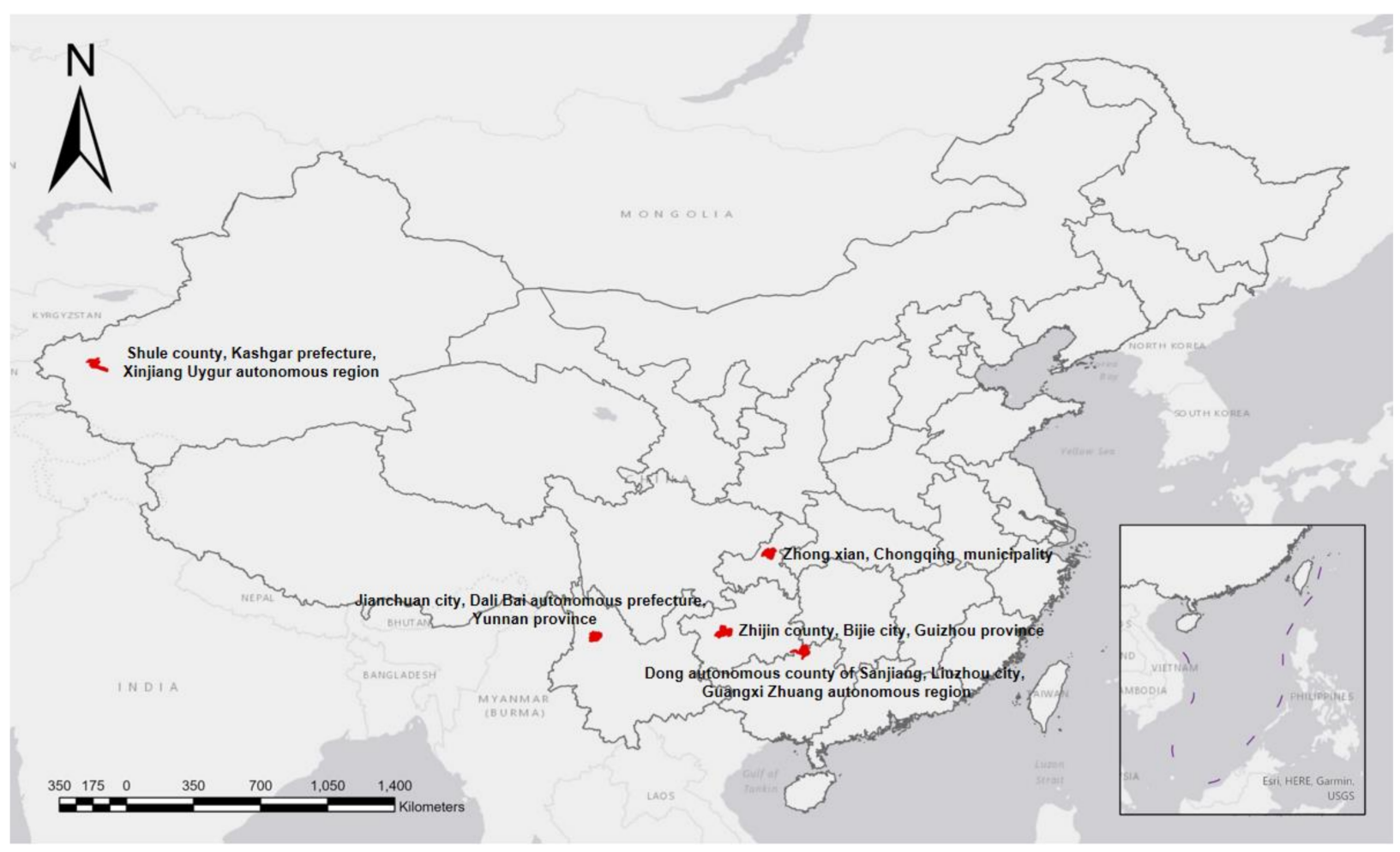

Figure 1. Locations of provinces where the study was conducted.

\subsection{Survey Method}

From September to December 2018, the researchers visited the 60 selected schools and asked students to fill out an anonymous questionnaire by themselves. Researchers explained the survey questions to the students before conducting the survey, checked their answers for completeness, and examined their hand hygiene and fingernail length when collecting the questionnaires. The questionnaire included questions about the students' demographics, hygiene knowledge, hygiene practices, school sanitation conditions, and whether they had had diarrhea in the 3 months prior to the survey period. All of the 2330 questionnaires distributed were returned and deemed valid. The teachers and students fully understood the survey content and agreed to participate in the survey, which was agreed on by the parents of the students before the survey. During the survey, the teachers were present in the whole process, and obtained the relevant health knowledge, attitude and behavior information through the students' self-administered questionnaire. The whole process did not cause any harm to the students, and they exchanged ideas. There is no risk to the target. Before the investigation, we strictly implemented the requirements of informed consent in the process of investigation. Through the review of information and on-site investigation according to the ethical review provisions, the project met the ethical requirements, and it has been proved by National Center for Rural Water Supply Technical Guidance, Chinese Center for Disease Control and Prevention.

\subsection{Quality Control}

The research subjects were randomly selected in strict accordance with related technical procedures. The questionnaire was subjected to expert consultation and pre-testing. The research team, which consisted of professional technicians, answered any questions raised by the students on-site and examined the completeness of their responses. Double data entry and real-time validation were used to ensure the accuracy of the data. 


\subsection{Statistical Analysis}

The researchers used Microsoft Excel 2016 for data entry and sorting and the Statistical Package for Social Sciences (SPSS) v19.0 for statistical analysis. Quantitative variables are represented as means \pm standard deviations (SD). Grouped data are expressed as percentages. Univariate analysis was performed with Chi-square test for diarrhea, and multiple logistic regression analysis was conducted with statistically significant factors and some potentially significant factors in univariate analysis as independent variables. Adjusted odds ratios (ORs) and their 95\% confidence intervals (CIs) were computed. Twotailed tests were carried out at an alpha level of 0.05 .

\section{Results}

\subsection{Basic Information}

The 2330 respondents (aged $9.9 \pm 0.3$ ) years consisted of 461 students from Guangxi Zhuang autonomous region, 538 from Chongqing municipality, 465 from Guizhou province, 410 from Yunnan province, and 456 from Xinjiang Uygur autonomous region. Of these, 1151 students $(49.40 \%)$ were male, $1489(63.91 \%)$ were of a minority ethnicity, and 433 (18.58\%) were boarding students. Approximately 771 students $(33.09 \%)$ suffered from diarrhea in the 3 months leading up to the survey; $32.67 \%$ of students in this subset were male $(n=376)$ and $33.50 \%$ were female $(n=395)$. Among the diarrhea-afflicted students, 175 were from Guangxi Zhuang autonomous region, 88 from Chongqing municipality, 156 from Guizhou province, 175 from Yunnan province, and 177 from Xinjiang Uygur autonomous region, accounting for $37.96 \%, 16.36 \%, 33.55 \%, 42.68 \%$, and $38.82 \%$ of the total respondents from these regions, respectively. Approximately $24.02 \%(n=202)$ and $38.21 \%$ ( $\mathrm{n}=569)$ of students of Han and minority ethnicity, respectively, contracted diarrhea, and $35.57 \%(\mathrm{n}=154)$ and $32.53 \%(\mathrm{n}=617)$ of boarding and non-boarding students, respectively, were found to have had the disease. The demographic information is outlined in Table 1.

Table 1. Demographics of study participants $(\mathrm{N}=2330)$.

\begin{tabular}{lc}
\hline \multicolumn{1}{c}{ Demographics } & $\begin{array}{c}\text { Number of Subjects (\% Subject Population) } \\
\text { or Mean } \pm \text { SD }\end{array}$ \\
\hline Age (years) & $9.9 \pm 0.3$ \\
Sex & $1151(49.40)$ \\
Male & $1179(50.60)$ \\
Female & \\
Ethnic group & $841(36.09$ \\
Han people & $1489(63.91)$ \\
Ethnic minority & \\
Boarding & $433(18.58)$ \\
Yes & $1897(81.42)$ \\
No & \\
Region & $538(23.09)$ \\
Chongqing municipality & $465(19.96)$ \\
Guizhou province & $461(19.79)$ \\
Guangxi Zhuang autonomous region & $456(19.57)$ \\
Xinjiang Uygur autonomous region & $410(17.60)$ \\
$\quad$ Yunnan province &
\end{tabular}

\subsection{Univariate Logistic Regression Analysis}

A chi-square test was carried out to analyze factors that may contribute to the spread of diarrhea in students, such as their basic information, hygiene knowledge, hygiene practices, and school sanitation conditions. Of the four basic information factors, "ethnic group" and "region" were found to be statistically significant $(p<0.05)$. Approximately $38.21 \%$ of students of minority ethnicity had suffered from the disease, compared to $24.02 \%$ of students of Han ethnicity. The survey showed that $42.68 \%$ of students from Yunnan Province had suffered from diarrhea, and that this region had the highest diarrhea rate among all five provinces. 
Of the nine factors related to hygiene knowledge, only the factor of "whether students know that diarrhea can be transmitted through contaminated water" was statistically insignificant $(p>0.05)$. Students who did not possess adequate hygiene knowledge were more vulnerable to diarrhea than those who did possess such knowledge. Close to 50.38\% of students who did not know the "the right way to wash their hands," $47.19 \%$ of students who did not know that "open defecation may pollute water sources, damage environmental sanitation, and breed mosquitoes and flies," $46.54 \%$ of students who did not know that "hand washing can prevent diseases like diarrhea, dysentery, hepatitis A, and roundworm disease," $45.81 \%$ of those who were unaware of that "diarrhea can be prevented by washing hands before meals and after going to the toilet," and $45.78 \%$ of students who did not know that "dirty hands can transmit diseases" had all suffered from diarrhea in the 3 months leading up to the survey.

All of the 11 hygiene practice factors were found to be statistically significant $(p<0.05)$. Students who did not practice hygiene were more susceptible to the disease than those who did. The diarrhea rates were high among students who "did not wash vegetables and fruits every time before eating them raw" (52.16\%), those who "did not like health classes" (51.28\%), those who "did not wash hands before every meal" $(50.28 \%)$, those who "did not wash hands after every visit to the toilet at home" (49.60\%), those who "have drunk untreated water at school" (48.27\%), those who "have drunk untreated water at home" (47.53\%), and those who "did not wash hands after every visit to the toilet at school" $(47.53 \%)$.

With respect to the seven school sanitation factors, only the factor of "whether students have to queue for toilets" was statistically insignificant $(p>0.05)$. Students were more likely to contract diarrhea if they had "no hand washing or drinking water facilities at school," "had to queue for toilets at school," or reported that "the toilets at school were unsafe with flies" and "the toilets smelled bad." Approximately 52.33\% of students in "schools with unsafe toilets," $49.72 \%$ of those in "schools without hand washing facilities," and $48.22 \%$ of those in "schools without drinking water facilities" had suffered from diarrhea in the 3 months leading up to the survey.

All of the above results are outlined in Table 2.

Table 2. Univariate and multiple logistic regression analysis of factors associated with the risk of contracting diarrhea.

\begin{tabular}{|c|c|c|c|c|c|c|c|c|c|c|c|}
\hline \multirow[b]{2}{*}{ Item } & \multirow[b]{2}{*}{ Category } & \multicolumn{5}{|c|}{ Univariate Logistic Regression Analysis } & \multicolumn{5}{|c|}{ Multiple Logistic Regression Analysis } \\
\hline & & $\begin{array}{l}\text { Number of } \\
\text { Respondents }\end{array}$ & $\begin{array}{l}\text { Number of } \\
\text { Students Who } \\
\text { Contracted } \\
\text { Diarrhea }\end{array}$ & $\begin{array}{l}\text { Diarrhea } \\
\text { Rate (\%) }\end{array}$ & $\begin{array}{c}x^{2} \\
\text { Value }\end{array}$ & $\begin{array}{c}p \\
\text { Value }\end{array}$ & $\begin{array}{c}\beta \\
\text { Value }\end{array}$ & $S E$ & $\begin{array}{c}\text { Wald } \\
\chi^{2} \text { Value }\end{array}$ & OR $(95 \% \mathrm{CI})$ & $\begin{array}{c}p \\
\text { Value }\end{array}$ \\
\hline \multirow{15}{*}{$\begin{array}{c}\text { Basic } \\
\text { Inform-ation }\end{array}$} & Sex & & & & 0.184 & 0.668 & - & - & - & - & - \\
\hline & Male & 1151 & 376 & 32.67 & & & - & - & - & - & - \\
\hline & Female & 1179 & 395 & 33.50 & & & - & - & - & - & - \\
\hline & Ethnic group & & & & 48.909 & 0 & & & & & \\
\hline & Han people & 841 & 202 & 24.02 & & & & & & 1.00 & \\
\hline & Ethnic minority & 1489 & 569 & 38.21 & & & 0.040 & 0.150 & 0.071 & $1.04(0.78,1.40)$ & 0.790 \\
\hline & Boarding & & & & 1.472 & 0.225 & - & - & - & - & - \\
\hline & Yes & 433 & 154 & 35.57 & & & - & - & - & - & - \\
\hline & No & 1897 & 617 & 32.53 & & & - & - & - & - & - \\
\hline & Region & & & & 96.815 & 0.000 & & & & & \\
\hline & $\begin{array}{l}\text { Chongqing } \\
\text { municipality }\end{array}$ & 538 & 88 & 16.36 & & & & & & 1.00 & \\
\hline & $\begin{array}{l}\text { Guizhou } \\
\text { province }\end{array}$ & 465 & 156 & 33.55 & & & 0.357 & 0.187 & 3.627 & $1.43(0.99,2.06)$ & 0.057 \\
\hline & $\begin{array}{c}\text { Guangxi Zhuang } \\
\text { autonomous } \\
\text { region }\end{array}$ & 461 & 175 & 37.96 & & & 0.249 & 0.222 & 1.262 & $1.28(0.83,1.98)$ & 0.261 \\
\hline & $\begin{array}{c}\text { Xinjiang Uygur } \\
\text { autonomous } \\
\text { region }\end{array}$ & 456 & 177 & 38.82 & & & 0.153 & 0.232 & 0.432 & $1.17(0.74,1.84)$ & 0.511 \\
\hline & Yunnan province & 410 & 175 & 42.68 & & & 0.717 & 0.217 & 10.887 & $2.05(1.34,3.14)$ & $\underset{*}{0.001}$ \\
\hline
\end{tabular}


Table 2. Cont.

\begin{tabular}{|c|c|c|c|c|c|c|c|c|c|c|c|}
\hline \multirow[b]{2}{*}{ Item } & \multirow[b]{2}{*}{ Category } & \multicolumn{5}{|c|}{ Univariate Logistic Regression Analysis } & \multicolumn{5}{|c|}{ Multiple Logistic Regression Analysis } \\
\hline & & $\begin{array}{l}\text { Number of } \\
\text { Respondents }\end{array}$ & $\begin{array}{l}\text { Number of } \\
\text { Students Who } \\
\text { Contracted } \\
\text { Diarrhea }\end{array}$ & $\begin{array}{l}\text { Diarrhea } \\
\text { Rate (\%) }\end{array}$ & $\begin{array}{c}x^{2} \\
\text { Value }\end{array}$ & $\begin{array}{c}p \\
\text { Value }\end{array}$ & $\begin{array}{c}\beta \\
\text { Value }\end{array}$ & $S E$ & $\begin{array}{c}\text { Wald } \\
x^{2} \text { Value }\end{array}$ & OR $(95 \% \mathrm{CI})$ & $\begin{array}{c}p \\
\text { Value }\end{array}$ \\
\hline \multirow{24}{*}{$\begin{array}{l}\text { Hygiene } \\
\text { know-ledge }\end{array}$} & $\begin{array}{c}\text { Drinking } \\
\text { untreated water } \\
\text { is harmful to } \\
\text { health. }\end{array}$ & & & & 29.504 & 0.000 & & & & & \\
\hline & Aware & 1887 & 576 & 30.52 & & & & & & 1.00 & \\
\hline & Unaware & 443 & 195 & 44.02 & & & 0.032 & 0.127 & 0.064 & $1.03(0.81,1.33)$ & 0.801 \\
\hline & $\begin{array}{l}\text { Diarrhea can be } \\
\text { transmitted } \\
\text { through } \\
\text { contaminated } \\
\text { water. }\end{array}$ & & & & 0.959 & 0.328 & - & - & - & - & - \\
\hline & Aware & 973 & 311 & 31.96 & & & - & - & - & - & - \\
\hline & Unaware & 1357 & 460 & 33.90 & & & - & - & - & - & - \\
\hline & $\begin{array}{l}\text { Dirty hands can } \\
\text { transmit } \\
\text { diseases. }\end{array}$ & & & & 20.287 & 0.000 & & & & & \\
\hline & Aware & 2081 & 657 & 31.57 & & & & & & 1.00 & \\
\hline & Unaware & 249 & 114 & 45.78 & & & 0.113 & 0.155 & 0.539 & $1.12(0.83,1.52)$ & 0.463 \\
\hline & $\begin{array}{l}\text { The correct way } \\
\text { to wash hands. }\end{array}$ & & & & 19.036 & 0.000 & & & & & \\
\hline & Aware & 2197 & 704 & 32.04 & & & & & & 1.00 & \\
\hline & Unaware & 133 & 67 & 50.38 & & & 0.148 & 0.208 & 0.507 & $1.16(0.77,1.74)$ & 0.476 \\
\hline & $\begin{array}{c}\text { Hand washing } \\
\text { can prevent } \\
\text { diseases like } \\
\text { diarrhea, } \\
\text { dysentery, } \\
\text { hepatitis A, and } \\
\text { roundworm } \\
\text { disease. }\end{array}$ & & & & 13.944 & 0.000 & & & & & \\
\hline & Aware & 2171 & 697 & 32.11 & & & & & & 1.00 & \\
\hline & Unaware & 159 & 74 & 46.54 & & & 0.097 & 0.199 & 0.238 & $1.1(0.75,1.63)$ & 0.626 \\
\hline & $\begin{array}{c}\text { Feces can } \\
\text { transmit diseases } \\
\text { like roundworm } \\
\text { disease, } \\
\text { hookworm } \\
\text { disease, diarrhea, } \\
\text { and dysentery. }\end{array}$ & & & & 8.060 & 0.005 & & & & & \\
\hline & Aware & 1974 & 630 & 31.91 & & & & & & 1.00 & \\
\hline & Unaware & 356 & 141 & 39.61 & & & -0.093 & 0.141 & 0.434 & $0.91(0.69,1.20)$ & 0.510 \\
\hline & $\begin{array}{l}\text { Diarrhea can be } \\
\text { prevented by } \\
\text { washing fruits } \\
\text { before eating } \\
\text { them raw and } \\
\text { not drinking } \\
\text { untreated water. }\end{array}$ & & & & 26.466 & 0.000 & & & & & \\
\hline & Aware & 1605 & 477 & 29.72 & & & & & & 1.00 & \\
\hline & Unaware & 725 & 294 & 40.55 & & & 0.276 & 0.105 & 6.939 & $1.32(1.07,1.62)$ & $\underset{*}{0.008}$ \\
\hline & $\begin{array}{l}\text { Diarrhea can be } \\
\text { prevented by } \\
\text { washing hands } \\
\text { before meals and } \\
\text { after going to the } \\
\text { toilet. }\end{array}$ & & & & 41.238 & 0.000 & & & & & \\
\hline & Aware & 1876 & 563 & 30.01 & & & & & & 1.00 & \\
\hline & Unaware & 454 & 208 & 45.81 & & & 0.432 & 0.119 & 13.096 & $1.54(1.22,1.95)$ & $0 *$ \\
\hline
\end{tabular}


Table 2. Cont.

\begin{tabular}{|c|c|c|c|c|c|c|c|c|c|c|c|}
\hline \multirow[b]{2}{*}{ Item } & \multirow[b]{2}{*}{ Category } & \multicolumn{5}{|c|}{ Univariate Logistic Regression Analysis } & \multicolumn{5}{|c|}{ Multiple Logistic Regression Analysis } \\
\hline & & $\begin{array}{c}\text { Number of } \\
\text { Respondents }\end{array}$ & $\begin{array}{l}\text { Number of } \\
\text { Students Who } \\
\text { Contracted } \\
\text { Diarrhea }\end{array}$ & $\begin{array}{l}\text { Diarrhea } \\
\text { Rate (\%) }\end{array}$ & $\begin{array}{c}x^{2} \\
\text { Value }\end{array}$ & $\begin{array}{c}p \\
\text { Value }\end{array}$ & $\begin{array}{c}\beta \\
\text { Value }\end{array}$ & $S E$ & $\begin{array}{c}\text { Wald } \\
x^{2} \text { Value }\end{array}$ & OR $(95 \% \mathrm{CI})$ & $\begin{array}{c}p \\
\text { Value }\end{array}$ \\
\hline & $\begin{array}{c}\text { Open defecation } \\
\text { may pollute } \\
\text { water sources, } \\
\text { damage } \\
\text { environmental } \\
\text { sanitation, and } \\
\text { breed } \\
\text { mosquitoes and } \\
\text { flies. }\end{array}$ & & & & 8.310 & 0.004 & & & & & \\
\hline & Aware & 2241 & 729 & 32.53 & & & & & & 1.00 & \\
\hline & Unaware & 89 & 42 & 47.19 & & & -0.017 & 0.254 & 0.005 & $0.98(0.60,1.62)$ & 0.946 \\
\hline \multirow{24}{*}{$\begin{array}{l}\text { Hygiene } \\
\text { practices }\end{array}$} & $\begin{array}{l}\text { I never drink } \\
\text { untreated water } \\
\text { at school. }\end{array}$ & & & & 143.404 & 0.000 & & & & & \\
\hline & Yes & 1464 & 353 & 24.11 & & & & & & 1.00 & \\
\hline & No & 866 & 418 & 48.27 & & & 0.451 & 0.115 & 15.344 & $1.57(1.25,1.97)$ & $0 *$ \\
\hline & $\begin{array}{l}\text { I never drink } \\
\text { untreated water } \\
\text { at home. }\end{array}$ & & & & 131.024 & 0.000 & & & & & \\
\hline & Yes & 1459 & 357 & 24.47 & & & & & & 1.00 & \\
\hline & No & 871 & 414 & 47.53 & & & 0.432 & 0.115 & 14.111 & $1.54(1.23,1.93)$ & $0^{*}$ \\
\hline & $\begin{array}{l}\text { I wash } \\
\text { vegetables and } \\
\text { fruits every time } \\
\text { before eating } \\
\text { them raw. }\end{array}$ & & & & 47.016 & 0.000 & & & & & \\
\hline & Yes & 2075 & 638 & 30.75 & & & & & & 1.00 & \\
\hline & No & 255 & 133 & 52.16 & & & 0.293 & 0.158 & 3.419 & $1.34(0.98,1.83)$ & 0.064 \\
\hline & $\begin{array}{l}\text { I wash hands } \\
\text { before every } \\
\text { meal. }\end{array}$ & & & & 56.449 & 0.000 & & & & & \\
\hline & Yes & 1972 & 591 & 29.97 & & & & & & 1.00 & \\
\hline & No & 358 & 180 & 50.28 & & & 0.358 & 0.147 & 5.962 & $1.43(1.07,1.91)$ & $\underset{*}{0.015}$ \\
\hline & $\begin{array}{l}\text { I wash hands } \\
\text { after every visit } \\
\text { to the toilet at } \\
\text { school. }\end{array}$ & & & & 27.914 & 0.000 & & & & & \\
\hline & Yes & 2067 & 646 & 31.25 & & & & & & 1.00 & \\
\hline & No & 263 & 125 & 47.53 & & & 0.061 & 0.167 & 0.135 & $1.06(0.77,1.47)$ & 0.713 \\
\hline & $\begin{array}{l}\text { I wash hands } \\
\text { after every visit } \\
\text { to the toilet at } \\
\text { home. }\end{array}$ & & & & 34.800 & 0.000 & & & & & \\
\hline & Yes & 2078 & 646 & 31.09 & & & & & & 1.00 & \\
\hline & No & 252 & 125 & 49.60 & & & -0.068 & 0.174 & 0.153 & $0.93(0.66,1.31)$ & 0.696 \\
\hline & $\begin{array}{l}\text { I wash hands } \\
\text { with running } \\
\text { water and } \\
\text { soap/hand } \\
\text { sanitizer at } \\
\text { school. }\end{array}$ & & & & & & & & & & \\
\hline & Yes & 1583 & 471 & 29.75 & 24.826 & 0.000 & & & & 1.00 & \\
\hline & No & 747 & 300 & 40.16 & & & 0.014 & 0.117 & 0.015 & $1.01(0.81,1.28)$ & 0.903 \\
\hline & $\begin{array}{l}\text { I wash hands } \\
\text { with running } \\
\text { water and } \\
\text { soap/hand } \\
\text { sanitizer at } \\
\text { home. }\end{array}$ & & & & 28.771 & 0.000 & & & & & \\
\hline & Yes & 1793 & 542 & 30.23 & & & & & & 1.00 & \\
\hline & No & 537 & 229 & 42.64 & & & 0.040 & 0.121 & 0.111 & $1.04(0.82,1.32)$ & 0.739 \\
\hline
\end{tabular}


Table 2. Cont.

\begin{tabular}{|c|c|c|c|c|c|c|c|c|c|c|c|}
\hline \multirow[b]{2}{*}{ Item } & \multirow[b]{2}{*}{ Category } & \multicolumn{5}{|c|}{ Univariate Logistic Regression Analysis } & \multicolumn{5}{|c|}{ Multiple Logistic Regression Analysis } \\
\hline & & $\begin{array}{l}\text { Number of } \\
\text { Respondents }\end{array}$ & $\begin{array}{l}\text { Number of } \\
\text { Students Who } \\
\text { Contracted } \\
\text { Diarrhea }\end{array}$ & $\begin{array}{l}\text { Diarrhea } \\
\text { Rate (\%) }\end{array}$ & $\begin{array}{c}x^{2} \\
\text { Value }\end{array}$ & $\begin{array}{c}p \\
\text { Value }\end{array}$ & $\begin{array}{c}\beta \\
\text { Value }\end{array}$ & $S E$ & $\begin{array}{c}\text { Wald } \\
x^{2} \text { Value }\end{array}$ & OR $(95 \% \mathrm{CI})$ & $\begin{array}{c}p \\
\text { Value }\end{array}$ \\
\hline & $\begin{array}{l}\text { I like health } \\
\text { classes. }\end{array}$ & & & & 24.991 & 0.000 & & & & & \\
\hline & Yes & 2174 & 691 & 31.78 & & & & & & 1.00 & \\
\hline & No & 156 & 80 & 51.28 & & & 0.128 & 0.195 & 0.433 & $1.14(0.78,1.67)$ & 0.511 \\
\hline & $\begin{array}{l}\text { My hands are } \\
\text { clean. }\end{array}$ & & & & 24.465 & 0.000 & & & & & \\
\hline & Yes & 1848 & 566 & 30.63 & & & & & & 1.00 & \\
\hline & No & 482 & 205 & 42.53 & & & 0.042 & 0.122 & 0.121 & $1.04(0.82,1.33)$ & 0.728 \\
\hline & $\begin{array}{l}\text { I have long } \\
\text { fingernails. }\end{array}$ & & & & 6.786 & 0.009 & & & & & \\
\hline & No & 1789 & 567 & 31.69 & & & & & & 1.00 & \\
\hline & Yes & 541 & 204 & 37.71 & & & 0.117 & 0.118 & 0.985 & $1.12(0.89,1.42)$ & 0.321 \\
\hline \multirow{21}{*}{$\begin{array}{c}\text { School } \\
\text { sanitation }\end{array}$} & $\begin{array}{l}\text { School has hand } \\
\text { washing } \\
\text { facilities. }\end{array}$ & & & & 23.919 & 0.000 & & & & & \\
\hline & Yes & 2153 & 683 & 31.72 & & & & & & 1.00 & \\
\hline & No & 177 & 88 & 49.72 & & & 0.150 & 0.187 & 0.648 & $1.16(0.81,1.68)$ & 0.421 \\
\hline & $\begin{array}{c}\text { School has } \\
\text { drinking water } \\
\text { facilities. }\end{array}$ & & & & 22.259 & 0.000 & & & & & \\
\hline & Yes & 2133 & 676 & 31.69 & & & & & & 1.00 & \\
\hline & No & 197 & 95 & 48.22 & & & 0.351 & 0.171 & 4.239 & $1.42(1.02,1.99)$ & $\underset{*}{0.040}$ \\
\hline & $\begin{array}{l}\text { School toilets are } \\
\text { clean. }\end{array}$ & & & & 34.117 & 0.000 & & & & & \\
\hline & Yes & 1726 & 513 & 29.72 & & & & & & 1.00 & \\
\hline & No & 604 & 258 & 42.72 & & & 0.110 & 0.129 & 0.727 & $1.12(0.87,1.44)$ & 0.394 \\
\hline & $\begin{array}{l}\text { I have to queue } \\
\text { for toilets. }\end{array}$ & & & & 1.839 & 0.175 & & & & & \\
\hline & No & 1537 & 494 & 32.14 & & & & & & & \\
\hline & Yes & 793 & 277 & 34.93 & & & & & & & \\
\hline & Toilets smell bad. & & & & 94.187 & 0.000 & & & & & \\
\hline & No & 969 & 212 & 21.88 & & & & & & 1.00 & \\
\hline & Yes & 1361 & 559 & 41.07 & & & 0.404 & 0.120 & 11.341 & $1.5(1.18,1.89)$ & $\underset{*}{0.001}$ \\
\hline & $\begin{array}{l}\text { There are flies in } \\
\text { the toilets. }\end{array}$ & & & & 96.381 & 0.000 & & & & & \\
\hline & No & 1101 & 253 & 22.98 & & & & & & 1.00 & \\
\hline & Yes & 1229 & 518 & 42.15 & & & 0.290 & 0.113 & 6.550 & $1.34(1.07,1.67)$ & $\underset{*}{0.010}$ \\
\hline & $\begin{array}{l}\text { The toilets are } \\
\text { safe. }\end{array}$ & & & & 14.923 & 0.000 & & & & & \\
\hline & Yes & 2244 & 726 & 32.35 & & & & & & 1.00 & \\
\hline & No & 86 & 45 & 52.33 & & & 0.260 & 0.245 & 1.131 & $1.30(0.8,2.10)$ & 0.288 \\
\hline
\end{tabular}

* Indicates that the significance level in the multiple logistic regression analysis is $p<0.05$. OR: odds ratio; CI: confidence interval.

\subsection{Multiple Logistic Regression Analysis}

A history of diarrhea in the 3 months leading up to the survey was designated as the dependent variable ( $\mathrm{Y}: 0=$ No diarrhea, $1=$ Diarrhea), and the aforementioned 27 statistically significant factors were included in the unconditional logistic regression model for analysis. We found that students from Yunnan province were at greater risk of contracting diarrhea than those from Chongqing municipality (OR: 2.05, 95\% CI: 1.34, 3.14). Students who did not know that "diarrhea can be prevented by not drinking untreated 
water and by washing fruits before eating them raw" (OR: 1.303, 95\% CI: 1.063, 1.597) and did not know that "the disease can be prevented by washing hands before meals and after going to the toilet" (OR: 1.522, 95\% CI: 1.207, 1.920) were more susceptible to diarrhea than those who knew the above-stated facts. Students who "have drunk untreated water at school" (OR: 1.584, 95\% CI: 1.268, 1.978), "have drunk untreated water at home" (OR: $1.643,95 \%$ CI: 1.319, 2.048), and "did not wash hands before every meal" (OR: 1.490, 95\% CI: 1.120, 1.983) were at higher risk than those who followed the above-described hygiene practices. Diarrhea was more likely to affect students who attended schools with poorly maintained and dirty toilets (OR: 1.586, 95\% CI: 1.261, 1.995), toilets that had flies (OR: 1.383, 95\% CI: 1.114, 1.717), and inadequate drinking water facilities (OR: 1.407, 95\% CI: $1.009,1.962)$. The results of this analysis are outlined in Table 1.

\section{Discussion}

The results of our study show that $33.09 \%$ of the research subjects had suffered from diarrhea in the 3 months leading up to the survey. Victoria et al. conducted a survey in Mali on 4907 fourth-grade students [17] with an average age of 11 years who were subjected to WASH interventions and 4823 similarly aged students who were not subjected to such interventions; they found that $10.0 \%$ and $13.0 \%$ of the students, respectively, had contracted diarrhea in the week preceding the investigation. A study conducted by Emma et al. showed that $11 \%$ of 2082 students (fourth- and fifth-graders, aged 10 years on average) surveyed from 116 schools across eight states and regions in Myanmar had contracted diarrhea in the week leading up to the survey [18]. Mansour et al. discovered that $14.9 \%$ of 1064 children surveyed in Saudi Arabia had contracted the disease in the month preceding the survey [19]. Other studies have shown that $16.4 \%$ [20], $14.42 \%$ [21,22], and $12.1 \%$ of children under the age of 5 years in Ethiopia, Indonesia, and Tanzania [23], respectively, had contracted diarrhea in the 2 weeks preceding the study. All of the above-described studies focused on diarrhea in children under the age of 5 years and on a time period of 1 month before the survey. Mahmud et al. found that the intestinal parasite reinfection rates were $29 \%, 26 \%$, and $38 \%$ in children who had not been subjected to the hand washing intervention, children whose fingernails were not cut, and children who had been subjected to neither of the interventions, respectively [22]. These results are consistent with those of our study.

We found that the number of students suffering from diarrhea in Yunnan province was higher than that in the other four regions (1.6 times that in Chongqing municipality). Liu M et al. showed that residents in Yunnan province did not perform well when tested for basic knowledge about health, good health practices, and skills for maintaining health [24]. They also argued that ethnic minorities were in greater need of improvements in health literacy. A study conducted by Zhou Y et al. showed that between 2011 and 2013, there were 39,347 reports of diarrhea from Yunnan province, amounting to an average incidence rate of 28.34/100,000 [25]. Furthermore, the province saw an upward trend in reported cases during this 3-year period. These data imply that to prevent the spread of diarrhea and other contagious diseases among rural students, more targeted health education and effective WASH interventions should be implemented.

The univariate logistic regression analysis showed that eight of nine factors related to hygiene knowledge were statistically significant $(p<0.05)$, suggesting that health education plays a key role in preventing the spread of infectious diseases such as diarrhea. The multiple logistic regression analysis indicated that a lack of the knowledge that "diarrhea can be prevented by washing fruits before eating them raw and not drinking untreated water" and "diarrhea can be prevented by washing hands before meals and after going to the toilet" were the two major risk factors associated with the disease. Students who were unaware of these two health factors had 1.32 and 1.54 times the risk for diarrhea, respectively, when compared with those who knew about and practiced them. Intestinal pathogens responsible for diarrhea are mainly transmitted through contaminated food and water by the fecal-oral route. Therefore, simple interventions, such as drinking boiled water, are very effective against the spread of diarrhea $[14,26]$. The outbreak of 
COVID-19 has attracted increased attention to the fecal-oral transmission of diseases. Viruses present in patient feces can be transmitted to others through food from fields contaminated by feces, food exposed to contaminated water, or objects, hands, and flies contaminated by virus-laden feces [27]. Unwashed hands are a main entry point for both viruses and bacteria. The proper hand washing guidelines laid out by the World Health Organization involve the use of soap and water, and include six steps to ensure that hands are thoroughly washed [28]. Hand washing helps to reduce the overall biomass of microbes, thus preventing diarrhea and respiratory infections as well as reducing the odds of resultant absences from school [29].

This study found 11 factors related to hygiene practices that were all statistically significant, implying that good hygiene practices are key to the prevention of infectious diseases such as diarrhea. The multiple logistic regression analysis indicated that "have drunk untreated water at school", "have drunk untreated water at home", and having not "washed hands before every meal" are the three major risk factors associated with diarrhea. Students who exhibited the above three behaviors faced 1.57, 1.54, and 1.43 times the risk, respectively, of contracting the disease than those who followed good hygiene practices. An epidemiological study on an infectious diarrhea outbreak in a school in Zhenba county in central Shaanxi province, China, reported that the outbreak was mainly triggered by students drinking dirty water [30]. Xiao et al. reported that "never washing vegetables and fruits/washing them occasionally before eating them raw" and "never washing hands/washing hands occasionally before meals and after going to the toilet" contribute to significant risks of intestinal parasite infections [31].

In addition to the above, the availability of hand washing and drinking water facilities and the conditions of toilets at schools are important factors that determine students' risk of contracting diarrhea. The multiple logistic regression analysis showed that schools without adequate drinking water facilities, with ill-maintained and unclean toilets, or with fly-ridden toilets posed significant hidden dangers for students. Students from schools that met these conditions faced 1.42, 1.5, and 1.34 times the risk, respectively, of contracting diarrhea than those who studied at schools with better sanitation conditions. The sanitation conditions of schools play a significant role in the development of good hygiene practices, as most children learn about such practices during their formal education [32]. If hand washing and adequate drinking water facilities are unavailable at schools, students cannot wash their hands properly and are more likely to drink untreated water [33]. A study conducted by Hughes et al. with Pacific Island communities found that students attending schools without adequate drinking water supplies faced a risk of contracting helminthiasis that was four times as high as that of students in schools with good water supply systems [34]. Students lacking access to safe water and sanitation are highly susceptible to infectious diseases, gastrointestinal problems, neurocognitive disorders, and mental health problems [35]. Recent research shows that 51\% of students avoid using school toilets [36] because the toilets are either dirty or unsafe [37]. Holding in urine/stool for long periods of time increases the risk of incontinence, constipation, and urinary tract infections [18]. Students with access to clean toilets at schools are also $12.17 \%$ less likely to suffer from diarrhea than those who lack access to such facilities [18].

Through the analysis of this study, it is concluded that the knowledge of methods to maintain personal hygiene, general hygiene practices, and school sanitation are the three major risk factors that account for the spread of diarrhea among rural students from five western provinces of China. Moreover, the number of students suffering from diarrhea in Yunnan province was higher than that in the other four regions.

Based on the risk factors associated with contracting diarrhea in students, we propose the following four suggestions to limit spread of the disease. First, health education must be prioritized and WASH interventions must be applied to vulnerable groups and poverty-stricken areas, especially Yunnan. Second, health education programs should be implemented in schools to raise awareness about sanitation and disease prevention; the programs should address topics such as the routes of disease transmission and help 
students develop good hygiene practices to protect themselves. Third, elements of the WASH intervention, such as conducting sanitation-themed lectures and health classes, distributing promotional materials advocating good health practices, and helping students stop bad hygiene practices and adopt good ones, such as not drinking untreated water, washing hands before meals and after using the toilet, washing raw fruits thoroughly before eating them, trimming fingernails often, and washing hands with running water and soap/hand sanitizer should be systematically implemented. Fourth, the water supply and sanitation conditions in schools should be improved; this is key to preventing diseases and keeping students healthy. Schools should either provide safe and clean drinking water facilities for students or mandate that students bring safe drinking water from home to prevent them from drinking untreated water. Hand washing facilities must be available to allow students to wash their hands before meals and after using the toilets at school. Schools should renovate unsafe and unhygienic toilets and teach students how to use a toilet properly; at the very least, they should ensure that the toilets are cleaned regularly and do not have flies. However, it is the implementation of these recommendations that may present challenges. Health literacy co-design can be carried out in rural areas to use local wisdom to inform interventions for rural students to improve health and equity [15].

The limitations of this study are as follows: 1 . The investigation of diarrhea is not based on medical records, but based on the questionnaire, and the results may have memory bias. 2. This study did not include all age groups of primary school students, only including the fourth grade students. The extrapolation of the relationship between diarrhea rate and environmental health knowledge and behavior of primary school students in different age groups was limited. 3. Due to the special research group and limited by the privacy of the questionnaire, this study did not consider other potential confounding factors such as socioeconomics, demographics, and other environments.

\section{Conclusions}

The knowledge of methods to maintain personal hygiene, general hygiene practices, and school sanitation are the three major risk factors that account for the spread of diarrhea among rural students from five western provinces (municipalities and autonomous regions) of China. Therefore, to prevent such diseases and maintain health, it is important to integrate the multi sectoral resources by government departments, such as education, health, Poverty Alleviation Office, agriculture and other departments, to further guide the work related to school water and environmental sanitation, make full use of domestic project funds and UNICEF project funds to bundle implementation, complement each other and develop together in order to provide students with health education, help them develop good hygiene habits, ensure the provision of clean water at schools, and improve the overall school environments.

Author Contributions: Conceptualization, W.Y. and Y.-E.C.; methodology, W.Y. and Y.-E.C.; software, Y.-E.C.; validation, W.Y., Y.-E.C. and Y.-Z.F.; formal analysis, Y.-E.C. and Y.-Z.F.; investigation, W.Y. and Y.-E.C.; resources, W.Y. and Y.-E.C.; data curation, W.Y. and Y.-E.C.; writing-original draft preparation, Y.-E.C.; writing—review and editing, W.Y.; visualization, Y.-E.C.; supervision, W.Y.; project administration, W.Y.; funding acquisition, W.Y. All authors have read and agreed to the published version of the manuscript.

Funding: This research was funded by United Nations International Children's Emergency Fund (UNICEF).

Institutional Review Board Statement: The study was conducted according to the guidelines of the Declaration of Helsinki, and approved by National Center for Rural Water Supply Technical Guidance, Chinese Center for Disease Control and Prevention.

Informed Consent Statement: Informed consent was obtained from all subjects' parents involved in the study.

Conflicts of Interest: The authors declare no conflict of interest. 


\section{References}

1. World Health Organization. The Treatment of Diarrhea: A Manual for Physicians and Other Senior Health Workers; World Health Organization: Geneva, Switzerland, 2005.

2. Guerrant, R.L.; Hughes, J.M.; Lima, N.L.; Crane, J. Diarrhea in developed and developing countries: Magnitude, special settings, and etiologies. Rev. Infect. Dis. 1990, 12, S41-S50. [CrossRef]

3. Berkman, D.S.; Lescano, A.G.; Gilman, R.H.; Lopez, S.L.; Black, M.M. Effects of stunting, diarrhoeal disease, and parasitic infection during infancy on cognition in late childhood: A follow-up study. Lancet 2002, 359, 564-571. [CrossRef]

4. GBD 2016 Diarrhoeal Disease Collaborators. Estimates of the global, regional, and national morbidity, mortality, and aetiologies of diarrhoea in 195 countries: A systematic analysis for the Global Burden of Disease Study 2016. Lancet Infect Dis. 2018, 18, 1211-1228. [CrossRef]

5. Walker, C.L.; Black, R.E. Diarrhoea morbidity and mortality in older children, adolescents, and adults. Epidemiol. Infect. 2010, 138, 1215-1226. [CrossRef]

6. Wang, Y.H.; Hu, Y. Burden of diarrhoeal diseases in China and World Bank countries at different economic levels from 1990 to 2017. Mod. Bus. Trade Ind. 2020, 41, 80-84. (In Chinese)

7. Liu, T.T. Analysis of causes of death and burden of disease in school aged children. In Proceedings of the Healthy China-4th Asia-Pacific International Congress on Health Emergency and Rescue, Shenzhen, China, 31 March 2018. (In Chinese).

8. Xu, Y.; Yan, B.Q. Current situation and management countermeasures of public health emergencies in Chinese schools. Chin. J. Sch. Health 2013, 34, 513-515. (In Chinese)

9. Zhang, X.; Gu, X.; Wang, L. Spatiotemporal variations in the incidence of bacillary dysentery and long-term effects associated with meteorological and socioeconomic factors in China from 2013 to 2017. Sci. Total Environ. 2021, 755, 142626. [CrossRef] [PubMed]

10. Li, S.; Schmidt, A.M.; Elliott, S.J. Socioeconomic factors and bacillary dysentery risk in Jiangsu Province, China: A spatial investigation using Bayesian hierarchical models. Int. J. Environ. Health Res. 2020, 1-12. [CrossRef] [PubMed]

11. Vally, H.; McMichael, C.; Doherty, C.; Li, X.; Guevarra, G.; Tobias, P. The Impact of a School-Based Water, Sanitation and Hygiene Intervention on Knowledge, Practices, and Diarrhoea Rates in the Philippines. Int. J. Environ. Res. Public Health 2019, $16,4056$. [CrossRef]

12. Cairncross, S.; Hunt, C.; Boisson, S. Water, sanitation and hygiene for the prevention of diarrhoea. Int. J. Epidemiol. 2010, 39 (Suppl. 1), i193-i205. [CrossRef]

13. Curtis, V.; Cairncross, S. Effect of washing hands with soap on diarrhoea risk in the community: A systematic review. Lancet Infect. Dis. 2003, 3, 275-281. [CrossRef]

14. Wolf, J.; Hunter, P.R.; Freeman, M.C. Impact of drinking water, sanitation and handwashing with soap on childhood diarrheal disease: Updated meta-analysis and meta-regression. Trop. Med. Int. Health 2018, 23, 508-525. [CrossRef] [PubMed]

15. Anwar, W.A.; Mostafa, N.S.; Hakim, S.A. Health Literacy Co-Design in a Low Resource Setting: Harnessing Local Wisdom to Inform Interventions across Fishing Villages in Egypt to Improve Health and Equity. Int. J. Environ. Res. Public Health 2021, 18, 4518. [CrossRef]

16. United Nations Children's Fund. Water and Sanitation Project Newsletter. Available online: https://www.unicef.cn/reports/ wash-newsletter-2019-01 (accessed on 1 July 2021). (In Chinese).

17. Trinies, V.; Garn, J.V.; Chang, H.H.; Freeman, M.C. The impact of a school-based water, sanitation, and hygiene program on absenteeism, diarrhea, and respiratory infection: A matched-control trial in Mali. Am. J. Trop. Med. Hyg. 2016, 94, 1418-1425. [CrossRef] [PubMed]

18. Weaver, E.R.; Agius, P.A.; Veale, H. Water, sanitation, and hygiene facilities and hygiene practices associated with diarrhea and vomiting in monastic schools, Myanmar. Am. J. Trop. Med. Hyg. 2016, 95, 278-287. [CrossRef]

19. Al-Ghamdi, M.A.; Bentham, G.; Hunter, P.R. Environmental risk factors for diarrhea among male schoolchildren in Jeddah City, Saudi Arabia. J. Water Health 2009, 7, 380-391. [CrossRef]

20. Shine, S.; Muhamud, S.; Adanew, S.; Demelash, A.; Abate, M. Prevalence and associated factors of diarrhea among under-five children in Debre Berhan town, Ethiopia 2018: A cross-sectional study. BMC Infect. Dis. 2020, 20, 174. [CrossRef]

21. Komarulzaman, A.; Smits, J.; de Jong, E. Clean water, sanitation and diarrhea in Indonesia: Effects of household and community factors. Glob. Public Health 2017, 12, 1141-1155. [CrossRef]

22. Mahmud, M.A.; Spigt, M.; Bezabih, A.M.; Pavon, I.L.; Dinant, G.J.; Velasco, R.B. Efficacy of handwashing with soap and nail clipping on intestinal parasitic infections in school-aged children: A factorial cluster randomized controlled trial. PLoS Med. 2015, 12, e1001837. [CrossRef] [PubMed]

23. Edwin, P.; Azage, M. Geographical variations and factors associated with childhood diarrhea in Tanzania: A national population based survey 2015-2016. Ethiop. J. Health Sci. 2019, 29, 513-524. [CrossRef] [PubMed]

24. Liu, M.; Li, L.Q.; Pu, S.C.; Tang, S.D.; Yi, J.R.; Mei, L.J. Analysis on health literacy and its influencing factors among residents in 86 poverty-stricken counties and districts in Yunnan Province. Chin. J. Health Educ. 2020, 1, 13-19. (In Chinese) [CrossRef]

25. Zhou, Y.M.; Kan, B.; Xu, W. Epidemic status and surveillance of infectious diarrhea in yunnan province. Parasitoses Infect. Dis. 2014, 12, 179-183. (In Chinese)

26. Cohen, A.; Colford, J.M. Effects of Boiling Drinking Water on Diarrhea and Pathogen-Specific Infections in Low- and MiddleIncome Countries: A Systematic Review and Meta-Analysis. Am. J. Trop. Med. Hyg. 2017, 97, 1362-1377. [CrossRef] [PubMed] 
27. Arslan, M.; Xu, B.; El-Din, M.G. Transmission of SARS-CoV-2 via fecal-oral and aerosols-borne routes: Environmental dynamics and implications for wastewater management in underprivileged societies. Sci. Total Environ. 2020, 743, 140709. [CrossRef] [PubMed]

28. World Health Organization. WHO Guidelines on Hand Hygiene in Health Care; World Health Organization: Geneva, Switzerland, 2009. Available online: https:/ / www.ncbi.nlm.nih.gov/books/NBK144013/pdf/Bookshelf_NBK144013.pdf (accessed on 1 July 2021).

29. Mbakaya, B.C.; Lee, P.H.; Lee, R.L. Hand hygiene intervention strategies to reduce diarrhea and respiratory infections among schoolchildren in developing countries: A systematic review. Int. J. Environ. Res. Public Health 2017, 14, 371. [CrossRef] [PubMed]

30. Ping, L.I.; Rong-Qing, M.A.; Wang, X.J. Epidemiological investigation on an outbreak of infectious diarrhea in a school of Zhenba County of Shanxi Province. Occup. Health 2009, 25, 35. (In Chinese)

31. Li, X.X.; Chen, J.X.; Wang, L.X. Intestinal parasite co-infection among pulmonary tuberculosis cases without human immunodeficiency virus infection in a rural county in China. Am. J. Trop. Med. Hyg. 2014, 90, 106-113. [CrossRef]

32. Freeman, M.C.; Greene, L.E.; Dreibelbis, R. Assessing the impact of a school-based water treatment, hygiene and sanitation programme on pupil absence in Nyanza Province, Kenya: A cluster-randomized trial. Trop. Med. Int. Health 2012, 17, 380-391. [CrossRef]

33. Reeves, L.M.P.P.; Poore, M.R. School toilets: Facilitating hand hygiene? A review of primary school hygiene facilities in a developed country. J. Public Health 2012, 34, 483-488. [CrossRef]

34. Hughes, R.G.; Sharp, D.S.; Hughes, M.C. Environmental influences on helminthiasis and nutritional status among Pacific schoolchildren. Int. J. Environ. Health Res. 2004, 14, 163-177. [CrossRef]

35. Jasper, C.; Le, T.T.; Bartram, J. Water and sanitation in schools: A systematic review of the health and educational outcomes. Int. J. Environ. Res. Public Health 2012, 9, 2772-2787. [CrossRef] [PubMed]

36. Lundblad, B.; Hellström, A.L. Perceptions of school toilets as a cause for irregular toilet habits among schoolchildren aged 6 to 16 years. J. Sch. Health 2005, 75, 125-128. [CrossRef] [PubMed]

37. Vernon, S.L.B.; Hellstrom, A.L. Children's experiences of school toilets present a risk to their physical and psychological health. Child Care Health Dev. 2003, 29, 47-53. [CrossRef] [PubMed] 